$$
\left|\int_{\alpha}^{\beta} d[f(x) g(x)]-\int_{\alpha}^{\beta} g(x) d f(x)-\int_{\alpha}^{\beta} f(x) d g(x)\right|
$$

is less than $\epsilon$ multiplied by the total variation of $f(x)$ plus the total variation of $g(x)$. Hence this expression must be equal to 0 and therefore the formula for integration by parts is valid under the above hypotheses.

UNIVERSITY OF MiCHIGAN

\title{
A SET OF POSTULATES FOR BOOLEAN ALGEBRA
}

\section{BY SOLOMON HOBERMAN AND J. C. C. MCKINSEY}

1. A New Set of Postulates. In the development of a Boolean Algebra, Boole's Law of Development

$$
f(x)=f(1) x+f(0) x^{\prime}
$$

stands out as a basic relationship. This law is so all embracing that the question naturally arises, if this is set as a postulate, what postulates in addition to it are needed to define a Boolean Algebra? Using as undefined a class $K$ and the Sheffer stroke function, we shall show that, in addition to a form of Boole's Law, only two "trivial" postulates are required.

\section{Postulates.*}

I. $K$ contains at least two elements.

II. If $a$ and $b$ are elements of $K$, then $a / b$ is an element of $K$.

Definitions: $a^{\prime}=a / a, a \cdot b=a^{\prime} / b^{\prime}$, and $a+b=(a / b)^{\prime}$.

III. There exists in $K$ a unique element 0 , such that, if $f(x)$ is any function definable in terms of/and elements of $K$, we have, for any $x$ in $K$,

$$
f(x)=f\left(0^{\prime}\right) x+f(0) x^{\prime} .
$$

TheOREM 1. $0^{\prime \prime}=0$.

Proof: From III, and the preceding definitions, we have

$$
x=0^{\prime} x+0 x^{\prime}=\left[\left(0^{\prime} x\right) /\left(0 x^{\prime}\right)\right]^{\prime} ;
$$

in particular

* This is the smallest set of postulates for a Boolean Algebra yet given. 


$$
0=\left[\left(0^{\prime} 0\right) /\left(00^{\prime}\right)\right]^{\prime} .
$$

Thus there exists in $K$ an element 1 , such that

$$
0=1^{\prime} \text {. }
$$

From (1) above

and

$$
1=0^{\prime} 1+01 \text {, }
$$

$$
1=1^{\prime \prime} 1+1^{\prime} 1^{\prime} \text {. }
$$

Letting $f(x)=x^{\prime \prime} x+x^{\prime} x^{\prime}$ in III, we have

$$
x^{\prime \prime} x+x^{\prime} x^{\prime}=\left(0^{\prime \prime \prime} 0^{\prime}+0^{\prime \prime} 0^{\prime \prime}\right) x+\left(0^{\prime \prime} 0+0^{\prime} 0^{\prime}\right) x^{\prime} ;
$$

in particular

$$
1=1^{\prime \prime} 1+1^{\prime} 1^{\prime}=\left(0^{\prime \prime \prime} 0^{\prime}+0^{\prime \prime} 0^{\prime \prime}\right) 1+\left(0^{\prime \prime} 0+0^{\prime} 0^{\prime}\right) 1^{\prime} .
$$

This becomes

$$
1=0^{\prime \prime \prime} 1+0^{\prime} 1^{\prime},
$$

since letting $f(x)$ equal $x^{\prime \prime}$ and $x^{\prime}$ respectively in III yields

$$
x^{\prime \prime}=0^{\prime \prime \prime} x+0^{\prime \prime} x^{\prime}, \quad \text { and } \quad x^{\prime}=0^{\prime \prime} x+0^{\prime} x^{\prime} ;
$$

in particular

$$
0^{\prime \prime \prime}=0^{\prime \prime \prime} 0^{\prime}+0^{\prime \prime} 0^{\prime \prime}, \quad \text { and } \quad 0^{\prime}=0^{\prime \prime} 0+0^{\prime} 0^{\prime} .
$$

Letting $f(x)=0^{\prime \prime \prime} x+x^{\prime \prime} x^{\prime}$ in III, we have

$$
0^{\prime \prime \prime} x+x^{\prime \prime} x^{\prime}=\left(0^{\prime \prime \prime} 0^{\prime}+0^{\prime \prime \prime} 0^{\prime \prime}\right) x+\left(0^{\prime \prime \prime} 0+0^{\prime \prime} 0^{\prime}\right) x^{\prime} ;
$$

in particular

$$
1=0^{\prime \prime \prime} 1+1^{\prime \prime} 1^{\prime}=\left(0^{\prime \prime \prime} 0^{\prime}+0^{\prime \prime \prime} 0^{\prime \prime}\right) 1+\left(0^{\prime \prime \prime} 0+0^{\prime \prime} 0^{\prime}\right) 1^{\prime} \text {. }
$$

This becomes

$$
1=0^{\prime \prime \prime} 1+0^{\prime \prime} 1^{\prime}
$$

since

$$
0^{\prime \prime \prime}=0^{\prime \prime \prime} 0^{\prime}+0^{\prime \prime \prime} 0^{\prime \prime}, \text { and } 0^{\prime \prime}=0^{\prime \prime \prime} 0+0^{\prime \prime} 0^{\prime} \text {. }
$$

From III, $x^{\prime \prime}=0^{\prime \prime \prime} x+0^{\prime \prime} x^{\prime}$; thus $1^{\prime \prime}=0^{\prime \prime \prime} 1+0^{\prime \prime} 1^{\prime}$, and $1=1^{\prime \prime}$. Hence $1^{\prime}=1^{\prime \prime \prime}$ and $0=0^{\prime \prime}$.

Theorem 2. $x^{\prime \prime}=x$. 
Proof: $x^{\prime \prime}=0^{\prime \prime \prime} x+0^{\prime \prime} x^{\prime}=0^{\prime} x+0 x^{\prime}=x$.

From Theorem 1 and the definition of 1 , we have $0^{\prime}=1$, and thus

$$
0 / 0=0^{\prime}=1, \text { and } 1 / 1=1^{\prime}=0 .
$$

THEOREM 3. $1 / 0=0 / 1=1$.

Proof: From III and the definitions, we have

$$
\begin{aligned}
& 1=0^{\prime} 1+01^{\prime}=0 / 0+1 / 1=1+0=(1 / 0)^{\prime} ; \\
& 0=1 / 0 . \\
& 0^{\prime}=0^{\prime \prime} 0+0^{\prime} 0^{\prime}=1 / 1+0 / 0=0+1=(0 / 1)^{\prime} ; \\
& 0=0 / 1 .
\end{aligned}
$$

TheOREM 4. $0+0=0,1+0=1,0+1=1,1+1=1,00=0$, $10=0,01=0$, and $11=1$.

Proof: These equations follow immediately upon using the results of the preceding theorems in the definitions of + and .

In the following theorems the equations are obtained by letting $f(x)$ equal the left-hand side.

TheOREM 5. $1 x=x=x \quad 1=0+x=x+0$.

Proof: $\quad 1 x=\left(\begin{array}{ll}1 & 1\end{array}\right) x+\left(\begin{array}{ll}1 & 0\end{array}\right) x^{\prime}=1 x+0 x^{\prime}$,

$$
\begin{aligned}
x & =1 x+0 x^{\prime}, \\
x 1 & =\left(\begin{array}{lll}
1 & 1) x+(0 & 1
\end{array}\right) x^{\prime}=1 x+0 x^{\prime},
\end{aligned}
$$$$
0+x=(0+1) x+(0+0) x^{\prime}=1 x+0 x^{\prime} \text {, }
$$

$$
x+0=(1+0) x+(0+0) x^{\prime}=1 x+0 x^{\prime} ;
$$

since all five are equal to $1 x+0 x^{\prime}$, the theorem follows.

THEOREM 6. $0 x=x 0=0=x x^{\prime}$.

Proof:

$$
\begin{aligned}
0 x & =\left(\begin{array}{ll}
0 & 1
\end{array}\right) x+\left(\begin{array}{ll}
0 & 0
\end{array}\right) x^{\prime}=0 x+0 x^{\prime}, \\
x 0 & =\left(\begin{array}{ll}
1 & 0
\end{array}\right) x+\left(\begin{array}{ll}
0 & 0
\end{array}\right) x^{\prime}=0 x+0 x^{\prime}, \\
0 & =0 x+0 x^{\prime}, \\
x x^{\prime} & =\left(\begin{array}{lll}
1 & 1^{\prime}
\end{array}\right) x+\left(\begin{array}{ll}
0 & 0^{\prime}
\end{array}\right) x^{\prime}=0 x+0 x^{\prime} ;
\end{aligned}
$$

since all four are equal to $0 x+0 x^{\prime}$, the theorem follows.

Theorem 7. $1+x=x+1=1=x+x^{\prime}$.

Proof:

$$
\begin{aligned}
1+x & =(1+1) x+(1+0) x^{\prime}=1 x+1 x^{\prime} \\
x+1 & =(1+1) x+(0+1) x^{\prime}=1 x+1 x^{\prime}, \\
1 & =1 x+1 x^{\prime}, \\
x+x^{\prime} & =\left(1+1^{\prime}\right) x+\left(0+0^{\prime}\right) x^{\prime}=1 x+1 x^{\prime} ;
\end{aligned}
$$


since all four are equal to $1 x+1 x^{\prime}$, the theorem follows.

ThEOREM 8. $a x=x a$.

Proof: $\quad a x=\left(\begin{array}{ll}a & 1\end{array}\right) x+\left(\begin{array}{ll}a & 0\end{array}\right) x^{\prime}=\left(\begin{array}{ll}1 & a\end{array}\right) x+\left(\begin{array}{ll}0 & a\end{array}\right) x^{\prime}=x a$.

Theorem 9. $a+x=x+a$.

Proof: $\quad a+x=(a+1) x+(a+0) x^{\prime}=(1+a) x+(0+a) x^{\prime}$

$$
=x+a \text {. }
$$

Theorem 10. $x+b c=(x+b)(x+c)$.

Proof:

$$
\begin{aligned}
(x+b)(x+c) & =(1+b)(1+c) x+(0+b)(0+c) x^{\prime} \\
& =(1)(1) x+(b)(c) x^{\prime}=1 x+b c x^{\prime} \\
& =(1+b c) x+(0+b c) x^{\prime}=x+b c .
\end{aligned}
$$

THEOREM 11. $x b+x c=x(b+c)$.

Proof: $\quad x b+x c=(1 b+1 c) x+(0 b+0 c) x^{\prime}=(b+c) x+0 x^{\prime}$

$$
=(b+c) x=x(b+c) \text {. }
$$

The postulates we have given are known to be true in a Boolean Algebra, therefore they are necessary. We shall show that they are sufficient by showing that Huntington's postulates are derivable from them.

2. Huntington's Postulates and their Derivation. The following is Huntington's set of postulates; to each is appended a brief indication of its derivation from those of our set.

1. (a) If $a$ and $b$ are elements of $K$, then $a+b$ is an element of $K$. By definition, $a+b=(a / b)^{\prime}=(a / b) /(a / b)$; by Postulate I, if $a$ and $b$ are elements of $K, a / b$ is an element, and $a+b$ is an element.

(b) If $a$ and $b$ are elements of $K$, then $a \cdot b$ is an element of $K$. By definition, $a \cdot b=a^{\prime} / b^{\prime}=(a / a) /(b / b)$ is an element of $K$ as above.

2. (a) There exists an element 0 in $K$ such that $a+0=a$.

(b) There exists an element 1 in $K$ such that $a \cdot 1=a$.

\section{Theorem 5.}

3. (a) If $a, b, a+b, b+a$ belong to $K$, then $a+b=b+a$. Theorem 9.

(b) If $a, b, a b, b a$ belong to $K$, then $a b=b a$.

Theorem 8.

4. (a) If $a, b, c, b c, a+b c, a+b, a+c$, and $(a+b)(a+c)$ belong to $K$, then $a+b c=(a+b)(a+c)$.

Theorem 10. 
(b) If $a, b, c, b+c, a(b+c), a b, a c$, and $a b+a c$ belong to $K$, then $a b+a c=a(b+c)$.

Theorem 11.

5. If 0 and 1 exist and are unique, then for every element a belonging to $K$ there exists an element $a^{\prime}$ in $K$ such that $a+a^{\prime}=1$ and $a a^{\prime}=0$.

Theorems 1, 6, and 7 .

6. There are at least two distinct elements in $K$.

Postulate I.

3. A Two Element Boolean Algebra. A set of postulates for a two element Boolean Algebra can be obtained by changing Postulate I to: " $K$ contains two and only two elements."

4. Independence Examples:

1. $K=\{\alpha\}, \quad \alpha / \alpha=\alpha$.

2. $K=\{0, \beta\}, \quad$\begin{tabular}{l|llll} 
& \\
\hline & & $\beta$ & $\gamma$ & $\delta$ \\
\hline & $\gamma$ & $\gamma$ & $\beta$ & 0 \\
$\gamma$ & $\gamma$ & $\gamma$ & 0 & 0 \\
& $\delta$ & 0 & $\beta$ & 0 \\
0 & 0 & 0 & 0
\end{tabular}.

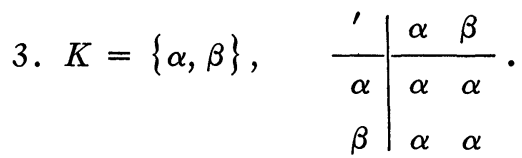

New YoRk University 